\title{
Erratum to: Antibiotic Resistance in Airborne Bacteria Near Conventional and Organic Beef Cattle Farms in California, USA
}

\author{
Helen M. Sanchez • Cristina Echeverria • Vanessa Thulsiraj • Amy Zimmer-Faust • \\ Ariel Flores • Madeleine Laitz • Gregory Healy • Shaily Mahendra • Suzanne E. Paulson • \\ Yifang Zhu $\cdot$ Jennifer A. Jay
}

Published online: 5 September 2016

(C) Springer International Publishing Switzerland 2016

\section{Erratum to: Water Air Soil Pollut \\ DOI 10.1007/s11270-016-2979-8}

Due to an error in the typesetting process, the family name of the first author Helen M. Sanchez was incorrectly written as "Sancheza".

The online version of the original article can be found at doi:10.1007/s11270-016-2979-8.

H. M. Sanchez • C. Echeverria • V. Thulsiraj •

A. Zimmer-Faust · A. Flores · M. Laitz • G. Healy •

S. Mahendra $\cdot$ S. E. Paulson $\cdot$ Y. Zhu $\cdot$ J. A. Jay $(\square)$

Department of Civil and Environmental Engineering, University of

California Los Angeles, Los Angeles, CA 90095, USA

e-mail: jjay@seas.ucla.edu

C. Echeverria

e-mail: cecheverria@ucla.edu

V. Thulsiraj

e-mail: vthulsiraj@msmu.edu

A. Zimmer-Faust

e-mail: amityzf@ucla.edu

A. Flores

e-mail: Ariel@smithemerylabs.com
M. Laitz

e-mail: mlaitz@mit.edu

G. Healy

e-mail: ghealy@andrew.cmu.edu

S. Mahendra

e-mail: mahendra@seas.ucla.edu

S. E. Paulson

e-mail: Paulson@atmos.ucla.edu

Y. Zhu

e-mail: yifang@ucla.edu 